\title{
Self-Collection of Antepartum Anogenital Group B Streptococcus Cultures
}

\author{
Peter G. Torok, $M D$, and Jan R. Dunn, $M D$
}

Background: Group B streptococcus (GBS) from the maternal perineum is a leading cause of serious neonatal infection. Recommendations by the Centers for Disease Control and Prevention include cultures from anogenital swabs on all pregnant women between 35 and 37 weeks' gestation. This study examines whether a significant difference in test results exists between GBS cultures from swabs done by physicians and those done by patients, and whether patients prefer to perform the swab themselves, with the potential of enhancing patient satisfaction and decreasing utilization of health care resources.

Metbods: Study design was a comparison of 250 obstetrics patients attending a military community hospital family medicine clinic. Patients were assigned on an alternating basis to perform an anogenital culture swab before or after the physician performed a swab. Patients were designated to complete a preference survey before or after the swabs were performed. Descriptive statistics, chi square, and McNemar's test corrected for multiple comparisons, and the Kruskal-Wallis test on effect of order on test outcome were used to analyze the data.

Results: Forty-two of 250 patients had positive cultures. There was no statistically significant difference between swab results done by patients and those done by physicians. There was no significant difference between results of first swabs and results of second swabs. Of the 250 patients 145 preferred self-collection. There was no statistically significant relation between preference and order of completion of the questionnaire.

Conclusions: Patient-collection technique is as accurate as physician collection. A majority of patients in this study population preferred self-collection of GBS swabs. (J Am Board Fam Pract 2000;13: 107-10.)

Group B streptococcus (GBS), or Streptococcus agalactiae, is a major cause of perinatal morbidity and mortality. GBS colonization of the anogenital area occurs in $10 \%$ to $30 \%$ of pregnant women, and $1 \%$ to $2 \%$ of infants born to colonized women will develop invasive infection during the first week of life (known as early-onset GBS infection). An estimated $6 \%$ of these infants will die from the disease. $^{1,2}$

Many strategies for the prevention of perinatal GBS infection have been proposed but have not been implemented consistently. ${ }^{3-5}$ Consequently, the Centers for Disease Control and Prevention (CDC) has published prevention guidelines that represent a consensus of experts and are endorsed by the American College of Obstetricians and Gy-

Submitted, revised, 21 October 1999.

From the Family Practice Service, Evans Army Community Hospital, Fort Carson, Colo. Reprints are not available.

The views expressed herein are those of the authors and do not necessarily reflect those of the United States Army Medical Department or the Department of Defense. necologists and the American Academy of Pediatrics. ${ }^{1,6,7}$ The $C D C$ recommendations base intrapartum chemoprophylaxis on intrapartum risk factors or results of prenatal anogenital cultures collected at 35 to 37 weeks' gestation. They recommend collecting a single culture swab or two separate swabs of the distal vagina and anorectum without performing pelvic examination or visualization of the cervix by speculum examination. They stipulate that specimens should be inoculated into selective broth medium. The CDC recommendations do not, however, specify by whom or in what setting (examination room or at home) the anogenital swab should be collected.

This study examines whether a statistically significant difference exists between results of GBS culture swabs collected by physicians and those collected by patients and whether patients prefer to perform the swab collection themselves. It also examines whether culture results from the second swab are the same as the results from the first swab for each patient, regardless of whether the physician or the patient collected the first swab. 


\section{Methods}

This study was conducted at Evans Army Community Hospital, Fort Carson, Colo. Family Practice Clinic pregnant patients of 35 to 37 weeks' gestation, aged 18 years and older, were informed of the study details and then elected to participate on a voluntary basis after signing informed consent. Their questionnaire responses were included in the patient preference data analysis. The patients were a diverse blend of Army soldiers and spouses of varying racial and ethnic backgrounds with high school and university educational levels. The study made no attempt to control for these characteristics. This study was approved by the Institutional Review Board of Brooke Army Medical Center, Fort Sam Houston, Tex.

Two anogenital swabs for GBS culture were collected in a clinic examination room on each participant during a routine prenatal visit between 35 and 37 week' estimated gestational age. One swab was collected by the physician, and the other was collected by the patient herself. Participants were assigned to physician-collection or self-collection of the first swab on an alternating basis. Each of the 14 participating physicians began the study by collecting the first swab on their initial participant. The next participant collected her own swab first. The physician collected the first swab on the subsequent participant, and so on, in an alternating fashion.

Each patient completed a questionnaire regarding her preference for self-collection or physician collection. Patients were selected to complete the questionnaire either before or after the swabs were collected based on whether the last digit of the social security number was an odd or even number. Each patient was given a reference diagram of the female perineum with written instructions for performing the swab collection.

A sterile cotton-tipped swab collection and transport system (Starplex Scientific, Ontario, Canada) was used to collect each culture. The swab was wiped around the vaginal introitus, then the anus, and placed into the transport medium. Each swab was coded so that laboratory personnel were blinded as to whether patient or physician had performed the collection. Cultures were incubated for 18 to 24 hours in selective broth medium (Lim broth). ${ }^{1,8}$ They were then subcultured onto blood agar plates ( $5 \%$ sheep blood and trypticase soy agar
Table 1. Group B Streptococcus Culture Results of Physician- and Patient-Collected Swabs for 250

Patients.

\begin{tabular}{lcr}
\hline & \multicolumn{2}{c}{ Patient Swab } \\
\cline { 2 - 3 } Physician Swab & Positive & Negative \\
\hline Positive & 32 & 7 \\
Negative & 3 & 208 \\
\hline
\end{tabular}

Significance: $P=.3438$.

base) and incubated for 24 to 48 additional hours. Colonies suspected to be GBS were confirmed by latex agglutination testing (PathoDx, Diagnostic Products Corporation, Los Angeles).

Results were reported electronically to the ordering physician. All positive cultures were considered to be true positive. Patients with one or both cultures positive for GBS were designated in their obstetric medical record as requiring intrapartum chemoprophylaxis according to the CDC guidelines.

In this study, the independent variables were collector (patient, physician) and order (first, second). The dependent variable was cell culture result (positive, negative). The hypothesis was that there is no relation between cell culture results and either collector or order. Descriptive statistics, chisquare, and McNemar's test corrected for multiple comparisons were used to analyze the data, which comprised seven variables. The Kruskal-Wallis test was used to evaluate the effect of order of swab collection on test outcome. The procedure for a 2 $X 2$ contingency test was used to perform a power analysis using a confidence level of $95 \%$ and a power of $80 \%$. 9,10

\section{Results}

Two hundred fifty patients participated, and 75 patients declined to participate in the study. Of the 250 women included in our study population, 42 (17\%) tested positive for GBS. There were no detected adverse effects of culture swabs collected by patients or physicians. Thirty-nine $(16 \%)$ of physician-collected and 35 (14\%) of patient-collected cultures were positive for GBS (Table 1). There was no statistically significant difference in culture results from swabs collected by physicians compared with those collected by patients $(P=$ .34). There was no statistically significant differ- 
ence in culture results between the first and second swabs regardless of who collected them $(P=1.00)$. One hundred forty-five of $250(58 \%)$ patients preferred self-collection. There was no statistically significant relation between preference for self-collection and the timing of questionnaire completion $(P>.05)$. Power analysis indicated that 240 subjects were needed to avoid the possibility of a type II error.

\section{Discussion}

Until a sufficiently sensitive rapid diagnostic test for GBS becomes generally available, anogenital cultures will remain an integral part of prevention strategies for perinatal GBS disease. ${ }^{11,12}$ Cultures collected within 5 weeks of delivery improve predictive value to nearly $100 \% .{ }^{13}$ Guidelines for prophylaxis based on obstetric risk factors do not address the $40 \%$ of early-onset GBS cases that occur in newborns of women without risk factors. ${ }^{14}$ For these reasons, the $\mathrm{CDC}$ recommendations include screening GBS cultures for all pregnant patients at 35 to 37 weeks' gestation.

Collecting anogenital swabs at 35 to 37 weeks' estimated gestational age intrudes on a woman's privacy when perineal examination is otherwise not routinely necessary. Clinician collection of swabs consumes appointment time and clinic resources. Patient self-collection of vaginal introitus and anorectal swabs has previously been shown to be accurate and reliable. ${ }^{15-17} \mathrm{~A}$ single swab of the anogenital area is as accurate as separate swabs of the vaginal introitus and anorectum. ${ }^{1}$ The use of a written patient instruction sheet facilitates self-collection of the swab. Because culture swabs may be placed in transport medium at environmental temperatures for up to 96 hours, ${ }^{7}$ self-collection of a single GBS swab at home or in the clinic is a feasible alternative to clinician-collection and a means of conserving health care resources while enhancing patient privacy.

Our study of a military community reveals an estimated prevalence of GBS colonization of $17 \%$, which correlates well with the national prevalence of $10 \%$ to $30 \%$ cited by the CDC. Our findings of no significant difference between patient- and physician-collected cultures and a preference for selfcollection correlate with the findings of a similar but unblinded and nonrandomized study. ${ }^{16,17}$ That study included separate swabs of the vagina and anorectum collected first by the patient then by a nurse who collected the swabs on all the participants (four swabs per patient). Our alternating collection sequence, the use of a single anogenital swab instead of separate vaginal introitus and anal swabs, and the participation of multiple clinicians differed from that study but led to similar results.

We also questioned whether the second swab for each patient would yield fewer positive GBS cultures after the first swab was collected, perhaps due to a reduced presence of the organism. Fewer positive GBS cultures would cause increased falsenegative results. There was no statistically significant difference between the results of swabs collected first and the results of those collected second.

Patient preference for self-collection did not seem to be influenced by the timing of questionnaire completion relative to the collection of the swabs. More than $50 \%$ of the patients preferred self-collection. Of the patients who refused to participate, the most frequently cited reason was not wanting to bother with the extra time needed to perform repeated swab collection. The next most frequent reason was concern about collecting the swab accurately. This anecdotal information was received from the participating physicians and was not solicited directly from the patients. Although we did not specifically examine refusal to participate, there was no apparent common characteristic of patients who declined to take part in the study.

This study involved a military community with a diverse blend of ethnic, social, and racial backgrounds that could be generalized to other populations, although a military community study population could also be considered to have biased the results of this study. Future studies could evaluate other communities' perceptions to include reasons for refusal in the patient preference questionnaire. Another potential bias of this study could be the individual physician's attitude or bedside manner toward screening for GBS and how it might have affected each patient's willingness to participate.

In conclusion, the findings of our study suggests self-collection of GBS cultures is an accurate and potentially cost-saving alternative for implementing the CDC screening guidelines for perinatal GBS colonization. Because a small majority of patients preferred collecting the culture swab themselves, pregnant patients could be given that option 
either in the clinic or at home during their 35 th to 37 th week of gestation.

The authors thank John A. Ward, PhD, Research Physiologist, Department of Clinical Investigation, Brooke Army Medical Center, Fort Sam Houston, Texas, for his assistance with the statistical analysis of the data.

\section{References}

1. Prevention of perinatal group B streptococcal disease: a public health perspective. Centers for Disease Control and Prevention. MMWR Morb Mortal Wkly Rep 1996;45(RR-7):1-24.

2. Zangwill KM, Schuchat A, Wenger JD. Group B streptococcal disease in the United States, 1990: report from a multistate active surveillance system. MMWR Morb Mortal Wkly Rep CDC Surveill Summ 1992;41(SS-6):25-32.

3. Rouse DJ, Goldenberg RL, Cliver SP, Cutter GR, Mennemeyer ST, Fargason CA Jr. Strategies for the prevention of early-onset neonatal group B streptococcal sepsis: a decision analysis. Obstet Gynecol 1994;83:483-94.

4. Mercer BM, Ramsey RD, Sibai BM. Prenatal screening for group B streptococcus. I. Impact of antepartum screening on antenatal prophylaxis and intrapartum care. Am J Obstet Gynecol 1995;173(3 Pt 1): 837-41.

5. Whitney CG, Plikaytis BD, Gozansky WS, Wenger JD, Schuchat A. Prevention practices for perinatal group B streptococcal disease: a multi-state surveillance analysis. Neonatal Group B Streptococcal Disease Study Group. Obstet Gynecol 1997;89(1):2832.

6. Prevention of early-onset group B streptococcal disease in newborns. ACOG Comm Opin 1996;173: $1-8$.

7. Revised guidelines for prevention of early-onset group B streptococcal (GBS) infection. American
Academy of Pediatrics Committee on Infectious Diseases and Committee on Fetus and Newborn. Pediatrics 1997;99:489-96.

8. Baker CJ, Goroff DK, Alpert SL, Hayes C, McCormack WM. Comparison of bacteriologic methods for the isolation of group B streptococcus from vaginal cultures. J Clin Microbiol 1976;4:46-8.

9. Fleiss JL. Statistical methods for rates and proportions. New York: Wiley, 1973.

10. Kraemer HC, Thiemann S. How many subjects? Statistical power and analysis in research. Newbury Park, Calif: Sage Publications, 1987.

11. Baker CJ. Inadequacy of rapid immunoassays for intrapartum detection of group B streptococcal carriers. Obstet Gynecol 1996;88:51-5.

12. Yancey MK, Armer T, Clark P, Duff P. Assessment of rapid identification tests for genital carriage of group B streptococci. Obstet Gynecol 1992;80: 1038-47.

13. Boyer KM, Gadzala CA, Kelly PD, Burd LI, Gotoff SP. Selective intrapartum chemoprophylaxis of neonatal group B streptococcal early-onset disease. II. Predictive value of prenatal cultures. J Infect Dis 1983;148:802-9.

14. Gotoff SP, Boyer KM. Prevention of early-onset neonatal group B streptococcal disease. Pediatrics 1997;99:866-9.

15. Wiesenfeld HC, Heine RP, Rideout A, Macio I, DiBiasi F, Sweet RL. The vaginal introitus: a novel site for Chlamydia trachomatis testing in women. Am J Obstet Gynecol 1996;174:1542-6.

16. Mercer BM, Taylor MC, Fricke JL, Baselski VS, Sibai BM. The accuracy and patient preference for self-collected group B streptococcus cultures. Am J Obstet Gynecol 1995;173:1325-8.

17. Taylor MC, Mercer BM, Engelhardt KF, Fricke JL. Patient preference for self-collected cultures for group B streptococcus in pregnancy. J Nurse Midwifery $1997 ; 42: 410-3$. 